\title{
Simulation of Urban Spatial Expansion in Lianyungang Lei Cao
}

School of Urban and Environment, Huaiyin Normal University, Huai'an, Jiangsu, 223001

\begin{abstract}
By simulating the distribution situation of urban landuse, practical reference can be provided for the future social and economic development of Lianyungang city and effective ecological environment protection. The urban expansion simulation of Lianyungang region is done by using GeoSOS. By using the artificial neural network and cellular automata model, urban space pattern is simulated to find out the factors of urban driving force. The results indicate: (1) The city of lianyungang and its area are expanding fast. There are big differences of the speed and scale of urban expansion in different spatial positions; (2) Urban expansion mainly depends on the encroachment of sorrounding high-quality farmlands; (3) The main driving forces of urban expansion include the environment of approaching to the Yellow Sea and the high-speed economic development of Lianyungang city.
\end{abstract}

Keywords: Geographical simulation systems; Cellular automation; Artificial neural networks; Urban expansion

\section{连云港城市空间扩展模拟}

\author{
曹蕾
}

(淮阴师范学院城市与环境学院, 江苏淮安 223001)

摘要: 通过对城镇用地分布情况进行模拟, 可以为连云港市未来的社会经济发展和有效保护生态环境提供出实际的参考。 通过 GeoSOS 对连云港地区进行城市扩展模拟, 采用人工神经网络结合元胞自动机模型模拟城市空间格局, 挖掘城市驱动力 因素。结果表明: (1) 连云港的城市扩展很快, 城市面积扩展迅速, 在不同的空间位置上城市扩展的速度和规模有较大的 差异;（2）城市扩展主要依靠对周边的优质农田的侵占; （3）连云港市经济快速发展及临海的地理位置决定着城市扩展的 空间方向和速度, 是城市扩展主要的驱动力。

关键词: 地理模拟系统; 元胞自动机; 人工神经网络; 城市扩展

中图分类号: TP751.1 文献标志码: A

\section{引言}

进入 21 世纪以来, 我国城市迎来了蓬勃的发展势头, 城市面积不断扩张, 城市经济与社会发展水平 表现出了持续的上升势头。然而, 随着城市化进程不断加快, 城市外延式盲目扩张、耕地大量占用等问题 影响了城市社会、经济和环境的发展。如何采取合理措施应对急剧变化的城市土地利用情况，明确城市未 来的发展方向并且实现城市化的可持续发展, 受到越来越多的关注 [1]。国内外许多学者通过不同方法对 城市扩张进行研究, 主要利用 GIS 空间分析技术、RS 与元胞自动机（Cellular Automata, CA）模型等。 元胞自动机具备很强的空间运算能力, 故而常用于自组织系统演变过程的研究, 可以通过局部转换规则来 模拟具有时空特征的复杂动态系统 [2]。近年来, 黎夏等人提出地理模拟优化系统 (Geographical Simulation and Optimization System, GeoSOS）的概念、研究内容和实现方法, 从而提供一种地理过程 分析与空间探索的有效工具 [3]。该系统在同一环境中对多个常用的 CA 模型加以整合, 具有较好的应用效 果 [4]。本文基于 GeoSOS 研究连云港市城市空间形态演变, 采用人工神经网络结合元胞自动机模型作为理 论模型, 将市中心、镇中心、铁路和主要公路等的距离作为区位因素空间距离约束条件, 将地形因子如坡 度和禁止城市化区域作为区位因素全局限制约束条件，对连云港市 2003-2009 年城市空间扩张进行精确模 
拟，并展开精度验证及成果分析，从而为城市规划提供决策支持。

\section{1 研究方法}

\section{1 地理模拟优化系统（GeoSOS）}

本文选用地理模拟优化系统 (GeoSOS) 为主要工作平台, 研究了连云港地区的城市扩展模拟。黎夏、 刘小平等人提出了地理模拟系统的理论框架, 可弥补常规 GIS 在动态过程模拟分析方面的不足 ${ }^{[5]}$, 并将多 智能体系统（Multi-agentSystems，MAS）、元胞自动机（Cellular Automata, CA）和群集智能（Swarm Intelligence, SI) 集成为地理模拟和空间优化系统 GeoSOS ${ }^{[6]}$ 。

元胞自动机模型是一种模拟复杂系统时空演变的有效工具, 可以反映出城市化演变规律, 常常被用于 城市空间扩张的模拟和预测 ${ }^{[7]}$ 以及城市规划情景分析 ${ }^{[89}$ 等等。目前基于 $\mathrm{CA}$ 的城市扩张模型有许多种, 每 种模型都有其自身的特点。GeoSOS 对多个城市元胞自动机模型加以整合, 如逻辑回归CA模型（Logistic-CA） ${ }^{[10]}$ 、神经网络 $\mathrm{CA}$ 模型 $(\mathrm{ANN}-\mathrm{CA})^{[11]}$ 、决策树-CA (DT-CA $)^{[12]}$ 等。

\section{2 ANN-CA 模型}

本文的主要研究方法为利用基于人工神经网络的 CA 模型进行模拟。人工神经网络算法有很多优点, 也可以解决多变量的情况, 十分适合运用到对复杂系统如土地利用系统的模拟之中。其算法主要如下:

(1)在网络中, 对于元胞 $\mathrm{x}$ 而言, 隐藏层中的神经元 $\mathrm{j}$ 从输入层接收到的信号可以表示为:

$$
\operatorname{net}_{j}(x, t)=\sum_{i} W_{i, j} S_{i}^{\prime}(x, t)
$$

(2) 将隐藏层中神经元 $\mathrm{j}$ 的激活函数设置为:

$$
\frac{1}{1+\exp \left[-\operatorname{net}_{j}(x, t)\right]}
$$

(3)元胞 $\mathrm{x}$ 的开发概率由输出层的输出结果和一个随机扰动项构成。前者可表示为:

$$
\sum_{j} W_{j} \frac{1}{1+\exp \left[-\operatorname{net}_{j}(x, t)\right]}
$$

(4)将随机扰动项表示为:

$$
\left\lfloor 1+(-\ln \gamma)^{\gamma}\right\rfloor
$$

(5)综上可得到元胞 $\mathrm{x}$ 在 $\mathrm{t}$ 时刻的开发概率:

$$
P_{d}^{\prime}(x, t)=\left[1+(-\ln \gamma)^{\alpha} \sum_{j} W_{j} \frac{1}{1+\exp \left[-\operatorname{net}_{j}(x, t)\right]}\right.
$$

\section{2 研究区概况和数据来源}




\section{1 研究区概况}

江苏省连云港市坐落在中国东部沿海、江苏省北部地区，位于陇海铁路东段，地处长江三角洲，同时 位于鲁中南丘陵和淮北平原的结合部。土地总面积约为 7500 平方千米, 水域面积约为 1760 平方千米, 市 区建成区面积约为 120 平方千米。连云港城市发展区主要由老城区（新浦区和海州区）和连云港东部滨海 地区（赣榆新区、海头镇、柘汪镇等）组成, 总面积约为 1806 平方千米。连云港是中国 14 个首批对外开 放的沿海城市之一, 不仅是我国中部沿海连接东西、沟通南北的区域性中心城市, 现代化的港口工业城市, 国际化的滨海旅游城市, 同时也是我国沿海中部的干线大港。连云港市地理位置非常优越, 并且近年来城 市化发展较快, 是江苏北部地区十分重要的经济、科技、文化中心, 故选取该城市进行扩展模拟具有较高 的应用价值。

\section{2 数据来源}

本文所需数据通过多年 TM 遥感影像处理和 GIS 数据库获取, 包括:

（1）期初期末模拟数据获取: 本研究采用连云港市不同年份的土地利用图作为模拟数据, 故所需土地 利用数据为 2003 年和 2009 年条带 121036 和 120036 的 TM 遥感影像;

（2）模型约束条件获取: 本研究通过坡度数据以及一系列空间距离变量来获取模型参数, 主要需要连 云港市规划和国土资源局提供的连云港市土地利用总体规划, 2003 年和 2009 年基础设施数据, 用来获取 市中心、镇中心、铁路、主要公路的矢量数据以及连云港市 DEM 影像数据。

\section{3 数据处理}

\section{1 数据源处理}

本研究选用 GeoSOS 作为平台, 主要需要输入 03、09 年土地利用图、坡度数据、空间距离变量。数据 源的处理过程大致如下:

（1）不同年份连云港市土地利用图: 首先获取 2003 年和 2009 年条带 121036 和 120036 的 TM 遥感影 像, 并在 ENVI 4.8 支持下, 将 03 年和 09 年连云港地区 TM 遥感影像进行辐射定标、大气校正等预处理, 结合监督分类和目视解译等方法, 对遥感影像进行分类, 得到土地利用数据。原 $\mathrm{TM}$ 影像分辨率太高, 行 列数太多, 为保证模型正确运行, 将 TM 影像在 ArcGIS 10.2 中适当调低分辨率, 重采样为 $100 \mathrm{~m} 。$ 经过栅 格计算和重分类, 最终得到的土地分类编码值为: 1 表示城市用地, 用黑色表示; 0 表示非城市用地, 用 白色表示。

（2）空间距离变量: 在 ArcGIS10.2 支持下, 结合连云港市规划和国土资源局数据、连云港市土地利 用总体规划, 基础设施数据, 获取市中心、镇中心、铁路、主要公路的矢量数据, 包括点、线等 shp 文件。 市中心、城镇中心、主要公路、铁路与已有城市用地的空间距离变量则通过 ArcMap 的 Spatial Analyst 中 的 Distance 工具计算得到。然后, 对不同年份的土地利用数据进行属性合成, 将城市变化数据进行导出, 采点生成点数据, 最终生成空间距离数据。

（3）坡度数据：由于连云港地区多山, 需要通过 $90 \mathrm{~m}$ 分辨率的 SRTM-DEM 数据, 来获取地形坡度信息。 将分辨率、投影坐标系和区域范围均与遥感影像统一并归一化为 0 1 。其中, 坡度数据以 0.25 为界进行重 分类, 从而得到坡度限制区。高于 0.25 的坡度设为 0 , 小于等于 0.25 的坡度设为 1 , 从而保证坡度低于 0.25 的土地可转变为城市用地。 
由于 GeoSOS 仅支持文本文件, 故需将上述数据进行 ASCII 二进制转换, 生成. $\mathrm{txt}$ 文件后输入软件进 行操作。为保证模型正确运行, 对除土地利用数据外的其他数据均进行了标准化, 将数值范围设为 $0 \sim 1$, 所有数据的分辨率统一为 $100 \mathrm{~m} \times 100 \mathrm{~m}$ 。

\section{2 研究过程}

通过 RS、GIS 获得有关数据, 处理后输入 GeoSOS, 利用空间数据对元胞自动机模型进行训练, 根据人 工神经网络算法自动获得模型参数, 并利用实际年份的分类结果进行模型纠正, 进一步设置有关模拟约束 参数。

主要的模拟参数包括元胞大小、元胞状态、元胞邻域、训练样本、元胞转换阈值、模拟终止条件和随 机干扰强度等。元胞自动机时空离散、状态有限, 各个元胞按一定规则分布在离散的元胞空间上, 时间变 量表现为等长时刻点, 状态明确并且是有限个。这些参数的设置需要根据实际需要来进行设置, 每一个参 数的变化都可能影响模拟结果出现较大的差异。在连云港地区的城市扩展模拟研究过程中，通过设定不同 的参数寻求模拟精度最高的元胞转换规则, 提高模拟的相对准确度。以上数据也对终止条件起到了控制, 从而获得最终模拟结果，并与期末真实土地利用图进行对比，进行精度评价和结果分析。

\section{4 空间约束条件}

\section{1 空间距离约束条件}

城市发展的许多决策判断准则可以由所得到的 “收益” 来衡量, 例如越接近城镇中心、交通越便利; 相反，所处位置越偏僻，城市发展所得到的 “收益”越小。“收益” 的具体大小可以由距离的梯度函数来 表达, 可称为 “空间可达性变量” ${ }^{[4]}$ 。这些空间因素对城市扩展的影响可以是正面的或者是负面的, 本文 所用空间距离约束条件由距市中心、镇中心、铁路、主要公路距已有土地利用的距离变量组成, 可以通过 RS 和 GIS 获取。

\section{2 全局限制约束条件}

在非城市用地向城市用地转换的过程中，除受到空间变量影响之外，一些其他的约束条件也会导致土 地利用的变化。例如森林, 重要耕地, 坡度地形不适合发展为城市用地的地区, 是在城市总体规划过程中 由于社会、自然等因素会被制约发展的一部分地区。本文主要选取地形坡度作为模型的全局约束条件。如 果某个元胞处于坡度大于 $25 \%$ 或者处于禁止建设区中，则认为该元胞不能向城市元胞发生转换。 


\section{5 结果分析}

\section{1 城市扩展及空间驱动力分析}

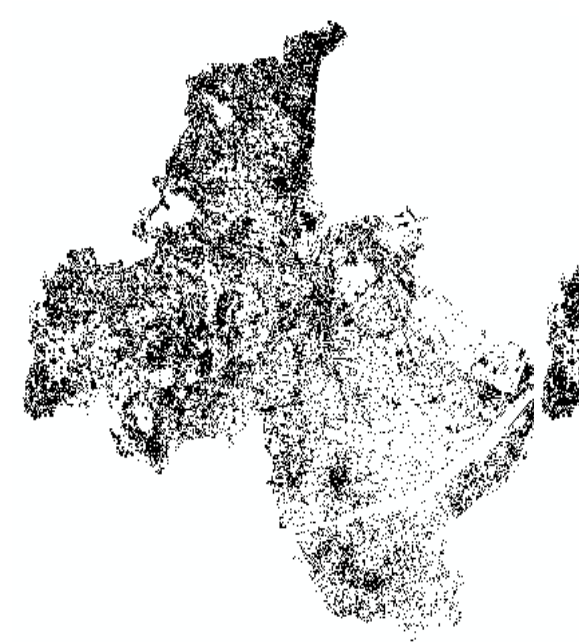

图 103 年土地利用图

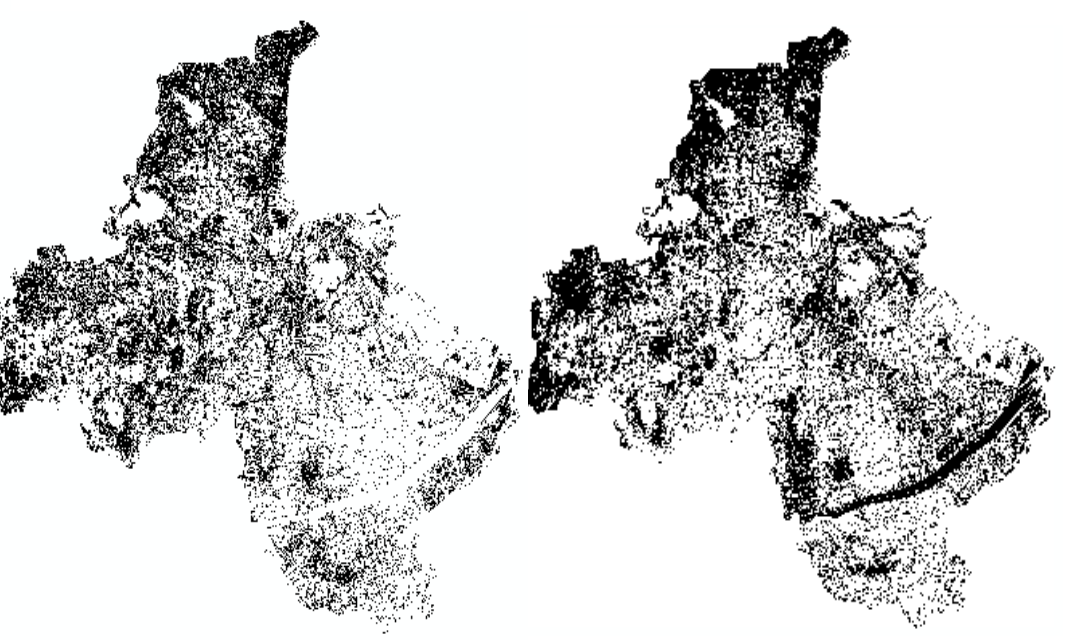

图 209 年土地利用模拟图

图 309 年土地利用图

ANN-CA 模型经过 GeoSOS 将 09 年土地利用模拟图（图 2) 与 09 年土地利用图（图 3）进行精度验证, 结果为 $62.9 \%$, 可较好地对连云港地区 03 年到 09 年的城市扩展进行模拟。通过对图 1、图 2、图 3 进行分 析, 连云港城市扩展主要依靠对耕地的侵占。从城市建设用地扩展情况来看, 主要为跳跃型开发城市新区。 城市建设用地在不同轴向上有不同扩展程度和规模, 呈现由初期的南北方向扩展过渡向后期的南北和东南 2 个方向扩展。因此需要处理好城市发展与农田减少之间的矛盾, 应积极采取耕地保护的措施, 防止发生 农业用地被乱占、占而不用等现象。

另外，城市化是复杂的地理现象，其发展归结为各种因素的综合作用，包括社会、自然因素 2 个方面 的因素 ${ }^{[13]}$ 。社会因素包括人口的增长, 经济的发展, 当地风俗文化的作用等。自然因素包括地形、气候、 生态环境等。结合连云港城市发展的相关政策和人口状况进行分析后可知, 连云港作为沿海开放城市, 在 国家对沿海城市发展的大力扶持下, 经济呈现稳步提升的良好态势, 良好的临海位置决定了经济因素是连 云港城市空间扩展最主要、最直接的动力。同时, 政策对连云港城市化的驱动作用也不可忽视, 连云港市 近年来致力于打造重点港口, 建设 “一心三局”、“一体两翼” 的城市布局和港口布局, 交通设施的发展 也向港口建设方向倾斜, 政策导向成为连云港城市发展的最根本驱动力。

\section{2 误差分析}

本文所用 $\mathrm{CA}$ 模型当迭代超过 1000 次，获得 62.9\%的较高精度。在 09 年模拟图（图 2) 与 09 年现状 图（图 3）比较中, 城市用地的扩展方向和规模都出现偏差, 南部地区出现了 “天窗” 现象。由于 ANN-CA 模型所用数据源较多, 来源不一, 本研究没有很好地进行标准统一, 也是导致模型精度不够高、模拟图和 真实图贴合不够精确的原因之一。另外, ANN-CA 模型中随机因素一定程度上影响了模型精度, 包括政府规 划产生的一些偏差。近年来连云港市区修路、建铁路等重大事件都会导致模型中空间变量产生变化, 从而 造成模型对城市演化的模拟失真。本文研究所用连云港区域数据皆为历史数据, 以此获取模型参数可视为 一种静态转换, 以上随机性变化模型无法做出对应的反应。 


\section{6 结论}

本文选取江苏省连云港市为研究区域，基于地理模拟优化系统（GeoSOS）提供的平台，通过人工神经 网络结合 $\mathrm{CA}$ 模型对连云港 03 年到 09 年城市建设用地扩展进行模拟分析, 并与实际用地情况进行了比较。 ANN-CA 模型综合考虑了研究区域的影响因子, 对模型及转换规则进行了限制, 模拟过程引入城市规划、政 府政策等因素，保证了技术过程的客观性、合理性。经过精度验证，模型可较好地对连云港地区 03 年到 09 年城市扩展进行模拟。通过对结果进行分析, 可了解连云港城市扩展主要形式为跳跃型开发城市新区。 城市建设用地在不同轴向上有不同扩展程度和规模, 呈现由初期的南北方向扩展过渡到后期的南北和东南 2 个方向扩展。通过数据分析, 连云港城市扩展主要侵占的是耕地。因此需要处理好城市发展与农田减少 之间的矛盾，应积极采取耕地保护的措施，防止发生农业用地被乱占、占而不用等现象。同时研究结果表 明, 利用 GeoSOS 结合遥感、GIS 数据研究城市扩展变化, 分析城市扩展驱动因素等是一种有效方法。

\section{7 致谢}

本研究受江苏高校哲学社会科学研究项目（2014SJB661）、淮安市科技计划项目（HAS2014022-3）和 全国统计科学研究项目（2016LZ39）的联合资助, 特此表示感谢。

\section{Acknowledgment}

This work was supported by The Project of Philosophy and Social Science Research in Colleges and Universities of Jiangsu Province (2014SJB661), The Science and Technology Plan Projects of Huaian (HAS2014022-3) and National Statistical Science Research Projects (2016LZ39). The author hereby expresses his gratitude.

\section{参考文献:}

[1] 刘盛和，吴传钧等. 基于 GIS 的北京城市土地利用扩展分析模式 $[J]$. 地理学报，2000(7)：407-416.

[2] 周成虎，孙战利，谢一春等. 地理元胞自动机研究 [M]. 北京：科学出版社， 2001.

[3] 陈逸敏，黎夏，刘小平等. 基于耦合地理模拟优化系统 Geo SOS 的农田保护区预警 [J]. 地理学报，2010，65(9)： $1137-1145$.

［4］管超，哈斯等. 基于GeoSOS 软件的城市扩展模拟--以旅顺口区为例 [J]. 测绘与空间地理信息, 2007, 35(3)：725727.

[5] 黎夏, 叶嘉安, 刘小平等. 地理模拟系统 [M]. 北京:科学出版社, 2007.

[6] 黎夏，刘小平，何晋强等. 基于耦合的地理模拟优化系统[J]．地理学报，2009，64 (8)：1009-1018.

[7] 黎夏, 刘小平. 基于案例推理的元胞自动机及大区域城市演变模拟 [J]. 地理学报, 2007，62 (10)：1097-1109.

[8] 刘小平, 黎夏. 人工免疫系统与嵌入规划目标的城市模拟及应用 [J]. 地理学报, 2008，63(8)：882-894. 999-1008.

[9］龙瀛，韩吴英，毛其智. 利用约束性 CA 制定城市增长边界 [J]。地理学报，2009，64 (8) : 872-895.

[10] Wu F. Calibration of stochastic cellular automata: The application to rural-urban land conversions [J]. International Journal of Geographical Information Science, 2002, 16(8): 795-818.

[11] 黎夏, 叶嘉安. 基于神经网络的单元自动机 CA 及真实和优化的城市模拟 [J]. 地理学报, 2002，57(2)：159-166.

[12] Li X, Yeh A G 0. Data mining of cellular automata's transition rules. International Journal of Geographical Information Science[J], 2004, 18: 723-744.

[13］再江，柯长青．连云港城市扩展及其驱动力分析 [J]．安徽农业科学，2007，35(3)：725- 727 . 


\section{References}

[1] Liu Chenghe, Wu Chuanjun and so on. Analysis of Urban Land Use Growth Model Based on GIS [J].Acta Geographica Sinica, 2000(7):407-416.

[2] Zhou Chenghu, Sun Zhanli, Xie Yichun and so on.Study on Geographical Cellular Automata [M].Beijing:Science Press,2001.

[3] Chen Yimin, Li Xia, Liu Xiaoping, and so on. Farmland Protection Warning Based on GeoSOS Optimization Coupled Simulation System [J].Acta Geographica Sinica, 2010, 65(9):1137-1145.

[4] Guan Chao,Ha Si,etc. Based on Urban Expansion Modeling of GeoSOS Software--a Case of Lvshunkou District [J].Geomatics \& Spatial Information Technology, 2007, 35(3):725-727.

[5] Li Xia, Ye Jia'an, Liu Xiaoping, and so on. Geographical Simulation Systems [M].Beijing: Science Press, 2007.

[6] Li Xia, Liu Xiaoping, He Jinqiang, and so on. Geographical Simulation and Optimization System Based on Coupling [J].Acta Geographica Sinica, 2009, 64(8):1009-1018.

[7] Li Xia, Liu Xiaoping. Urban Development Simulation of Based on Cellular Automata of Case-Based Reasoning [J].Acta Geographica Sinica, 2007, 62(10):1097-1109.

[8] Li Xia, Liu Xiaoping. Urban Simulation and Its Application of Artificial Immune System and Embedded Target [J].Acta Geographica Sinica, 2008, 63(8):882-894.999-1008.

[9] Long Ying, Han Haoying, Mao Qili. Using Binding CA to Establish Urban Growth Boundaries [J].Acta Geographica Sinica, 2009, 64(8):872-895.

[10] Wu F. Calibration of stochastic cellular automata: The application to rural-urban land conversions[J]. International Journal of Geographical Information Science, 2002, 16(8): 795-818.

[11] Li Xia, Ye Jia'an. Urban Simulation of Cellular Automaton CA and Real Optimization Based on Neural Network [J].Acta Geographica Sinica, 2002, 57(2):159-166.

[12] Li X, Yeh A G O. Data mining of cellular automata's transition rules. International Journal of Geographical Information Science [J], 2004, 18: 723-744.

[13] Ran Jiang, Ke Changqing. Analysis on Urban Expansion and Its Driving Force of Lianyungang [J].Journal of Anhui Agricultural Sciences, 2007, 35(3):725-727. 